\title{
Application of FBG Sensing Technology to Internal Deformation Monitoring of Landslide
}

\author{
Haiyou Peng $\mathbb{D}^{1,2}$, Bolin Chen $\mathbb{D}^{1,{ }^{1,2}}$ Ping Dong $\mathbb{D}^{1,2}$ Si chen $\mathbb{D}^{1,},{ }^{1,3}$ Yunping Liao, ${ }^{1,2}$ \\ and Qi Guo \\ ${ }^{1}$ Chongqing Institute of Geology and Mineral Resources, Chongqing 401120, China \\ ${ }^{2}$ Chongqing Engineering Research Center of Automatic Monitoring for Geological Hazards, Chongqing 401120, China \\ ${ }^{3}$ School of Environmental Studies, China University of Geosciences, Wuhan, Hubei 430074, China \\ Correspondence should be addressed to Haiyou Peng; penghaiyou@yeah.net
}

Received 22 July 2019; Revised 30 May 2020; Accepted 12 June 2020; Published 30 June 2020

Academic Editor: Carlo Rainieri

Copyright (c) 2020 Haiyou Peng et al. This is an open access article distributed under the Creative Commons Attribution License, which permits unrestricted use, distribution, and reproduction in any medium, provided the original work is properly cited.

Limited by geological survey methods, processes, and cost, it has long been a difficult thing to accurately detect the position of landslide slip surface and monitor the landslide internal deformation. Fiber Bragg grating (FBG) sensing technology has been widely used in geological engineering and geotechnical engineering due to its high-precision property. In this research, FBG sensing technology was applied to the monitoring of landslide internal deformation in Toudu, Chongqing, China. The in situ monitoring by FBG accurately determined the position of the landslide slip surface. Based on the relationship between fiber grating strain and deflection, the formula between landslide internal deformation and fiber grating strain was obtained, and the rationality of the formula was verified by the monitoring data of surface displacement. Finally, the internal deformation at the monitoring point of the Toudu landslide was calculated and the mechanism of the landslide was analyzed.

\section{Introduction}

China is one of the countries with frequent landslide disasters in the world. The early identification, investigation, and monitoring of landslides, as well as the survey, design, and construction management, have become an important part of geological work. Particularly, the correct identification of the position of slip surface (belt) is the most important work, as position identification plays a vital role in reasonably understanding the mechanism, scale, and degree of damage and formulating a reasonable disposal plan. However, at present, in most landslide engineering applications, the position of slip surface is usually acquired by obtaining the potential slip surface through field investigation and theoretical calculation. Due to the limitations of geological drilling processes, it is difficult to accurately locate the slip surface from drilled rock and soil samples. Besides, there are also many uncertainties in locating potential slip surfaces by theoretical calculation in the case of multiple uncertain slip surfaces. Further, the traditional inclinometer-based method also has many shortcomings for the monitoring of internal displacement and slip surface of a landslide.

Fiber Bragg grating (FBG) sensing technology is featured by high-precision, long-range, and long-term measurement and has been extensively applied to many fields. Since the first FBG was developed [1], the FBG sensing technology has been widely used in the stress, strain, health monitoring of structural engineering and geotechnical engineering, etc., for example, concrete structure monitoring $[2,3]$; monitoring of bridge prestress loss [4, 5]; health monitoring of dams, bridges, and tunnels [6-9]; pressure monitoring of tunnel's rock and soil [10]; lateral deformation monitoring of embankment soft soil [11]; and monitoring of basic engineering $[12,13]$.

At present, researchers have also started to apply FBG sensing technology to the monitoring of landslides and slopes [14-27] and have achieved good results. Investigations on landslide deformation and stability using optical fiber sensing technology have been extensively performed in previous researches, mainly focusing on obtaining the 
ground displacement of landslides through optical fibers and on researching the mechanism of internal deformation through laboratory experiments. While for investigations on both the internal deformation and deep displacement of the landslide, the optical fiber sensing technology is rarely adopted due to the difficulties in equipment installation, the complex field environments, and the uncontrollable boundary conditions. There is rare practical research on the in situ monitoring of landslide internal deformation. Taking the Toudu landslide, Chongqing as an example, this research applies FBG to the monitoring of landslide internal deformation. The feasibility of FBG in monitoring the deep displacement has been verified as well. And the position of the slip surface was determined. Additionally, relationship between the strain of the FBG and the internal displacement of the landslide has been established according to the straindeflection relationship of the fiber grating, which is further verified by the monitored data of the surface displacement. Therefore, the landslide internal deformation at the monitoring points was obtained. The research method and results in this paper provide a reference for locating the landslide slip surface as well as the theoretical research and engineering application of landslide internal deformation.

\section{Fundamentals}

2.1. FBG Sensing Technology. FBG sensing technology uses the sensitivity of fiber grating to light to form a spatial phase grating in the fiber core, as shown in Figure 1. When a broad-spectrum light beam enters the fiber grating, the wavelength that satisfies the fiber Bragg condition will produce a reflection, and the other wavelengths will continue to propagate through the fiber grating. When some physical quantity of the object to be measured changes to cause strain to the fiber grating, the center wavelength of the reflected light changes correspondingly, and the reflected light is received by the receiver. Finally, the change in the physical quantity of the measured object can be measured.

The direct external factors that cause wavelength drift are strain and temperature. When a fiber grating is used to monitor the physical quantities such as vibration, displacement, pressure, and inclination, it needs to be encapsulated in a specific structure that converts the physical quantities into another form, and the fiber grating in the structure will have a change in strain as a response to the change of external physical quantities, such as vibration and displacement. Then, the wavelength of reflected light will change in accordance with the change of external physical quantities. In summary, by detecting the wavelength variation, it is possible to acquire the change of external physical quantities, as illustrated in Figure 2.

2.2. Fundamental of the Monitoring of Landslide Internal Deformation with FBG. The monitoring of landslide internal deformation using FBG is mainly based on establishing the relationship between the mass points in a landslide and the deformation of fiber. As shown in Figure 3, when the rocksoil body inside the landslide is deformed, the fiber installed in the landslide body in advance will deform and generate an axial direction strain, causing a change in the wavelength of the reflected wave. Then, the wavelength information of the reflected wave is transferred to the ground receiver through the optical fiber, and the wavelength information recorded at different positions in the fiber is recorded. Through interpretation, we can know where the strain occurs and the value of the strain, thereby obtaining the deformation mechanism of the slip surface and the mass points inside the landslide body.

\subsection{Deflection Curve of Landslide Internal Deformation.} According to the fundamental described above, it is assumed that (1) the deformation of the landslide rock-soil body and the inclinometer is coordinated; (2) the mass points in the landslide are moving in a parallel direction to the landslide slope; (3) the holes for monitoring are drilled vertically; (4) the rock-soil body below the potential sliding surface will not deform, and (5) fiber grating (and the PVC pipe) is buried in the rock-soil body with the fiber bottom below the potential sliding surface. The deformation of fiber grating below the potential sliding surface is limited due to its strong cementation with the rock-soil body. Therefore, the fiber grating above the potential sliding surface could deform freely with the sliding slope, and the internal displacement of the landslide can be reflected by the strain of fiber grating.

Then, the formula for calculating the deflection curve of the landslide internal deformation can be established, as shown in Figure 4. Figure 4(a) is a simplified diagram of calculating the deflection curve of landslide internal deformation. Each grating segment can be regarded as a calculation unit, and the displacement of the mass points in the landslide is the sum of the displacements of all calculation units below. Figure 4(b) shows a single calculation unit. The spacing of the points in the fiber grating is the initial value $L_{0}$. After the landslide body slides, the mass point of the original landslide moves from position $\mathrm{A}$ to position $\mathrm{B}$. The movement of the mass point in the calculation unit is $L_{2 i}$, the spacing of the points in the grating segment is $L_{1}$, and the slope is $\gamma$.

According to the geometric relationship in Figure 4(b) and the cosine theorem, we have

$$
L_{1}^{2}=L_{2 i}^{2}+L_{0}^{2}-2 L_{2 i} L_{0} \cos \left(90^{\circ}-\gamma\right) .
$$

According to the strain relationship of the fiber grating, the length of the fiber deformed by the sliding body is expressed as

$$
L_{1}=L_{0}-\varepsilon_{i} L_{0} \text {, }
$$

where $\varepsilon_{i}$ is the strain of a point in the grating, $\varepsilon_{i}=\mu_{i} k\left(\mu_{i}\right.$ is the change in grating wavelength and $k$ is a constant related to the fiber grating material and strain).

The displacement of any mass point in the landslide is the sum of the displacements of all calculation units below it, given by

$$
L_{2}=\sum_{i=0}^{n} L_{2 i}
$$




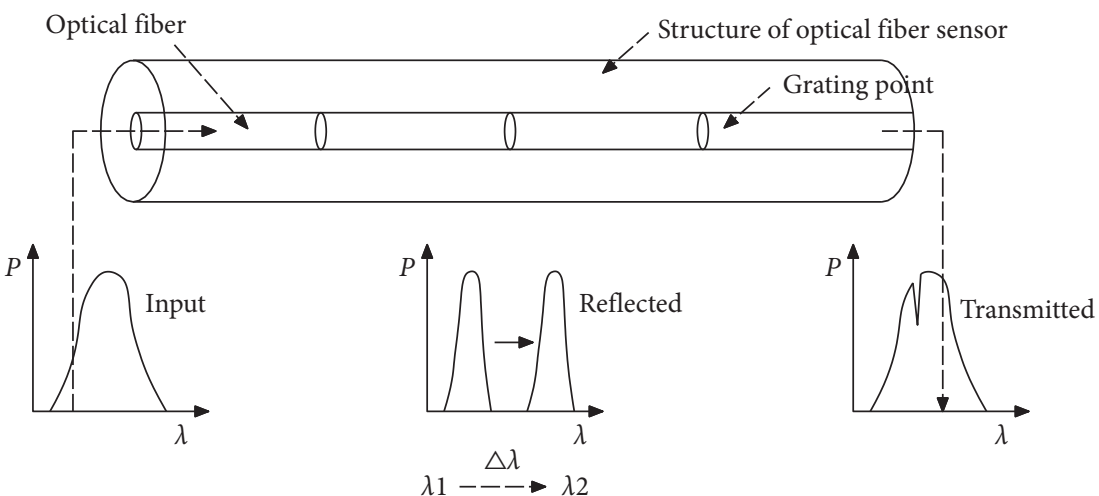

FIGURE 1: Illustration of FBG sensing technology.

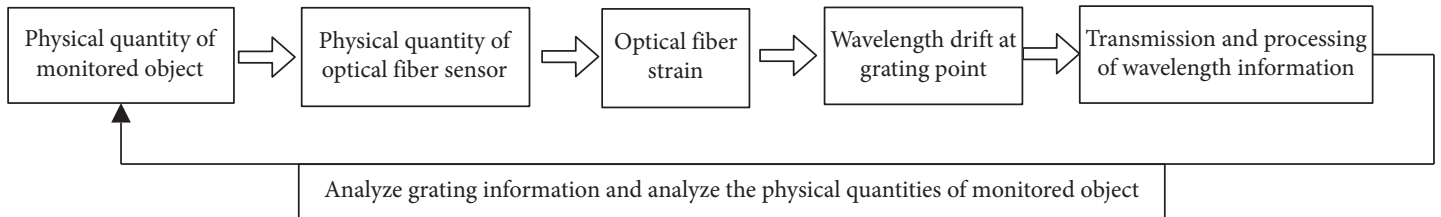

FIGURE 2: Fundamental of measuring external physical quantities using FBG sensing technology.

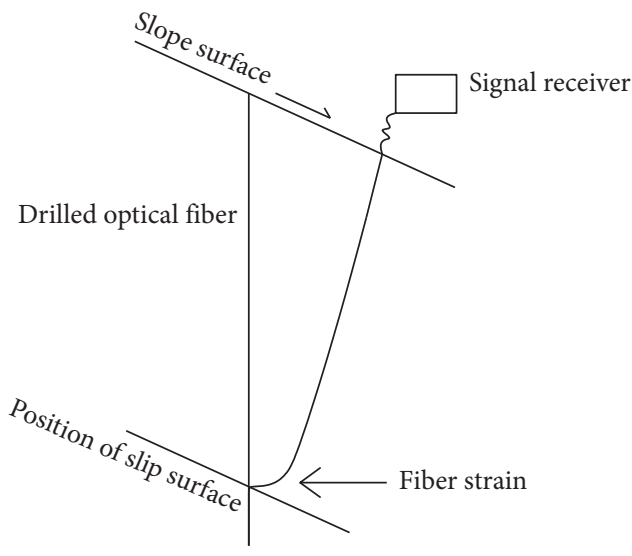

(a)

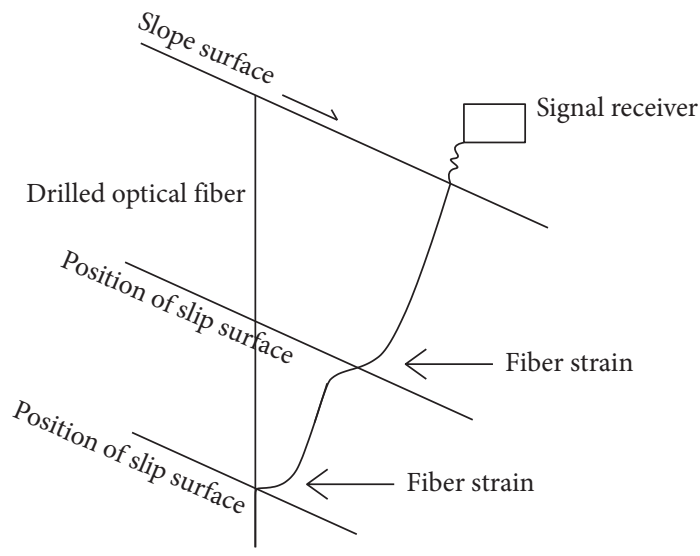

(b)

FIgURE 3: Fundamental of the monitoring of landslide internal deformation using fiber grating. (a) Single slip surface of landslide. (b) Multilayer slip surface landslide.

where $L_{2}$ is the displacement of measured point in the monitoring hole and $n$ is the number of fiber grating calculation units from the bottom to the monitoring point in the monitoring hole.

Substituting equation (2) into equation (1) to obtain $L_{2 i}$ which is then substituted into equation (3), we can obtain the formula for calculating the displacement of any mass point in the landslide with respect to the strain of the fiber grating:

$$
L_{2}=\sum_{i=0}^{n}\left[\cos \left(90^{\circ}-\gamma\right)-\sqrt{\left(\cos ^{2}\left(90^{\circ}-\gamma\right)-2 \varepsilon_{i}+\varepsilon_{i}^{2}\right)}\right] L_{0},
$$

where $L_{0}$ is the spacing of the points in the grating, $\gamma$ is the slope, and $\varepsilon_{i}$ is the strain at a point in the grating.

The displacement of measured point in the monitoring hole can be calculated by equation (4), where $\gamma$ (landslide slope) can be directly measured; $L_{0}$ (point spacing in the fiber grating) is the default value used as received from the factory; $n$ is the number of grating points that equidistantly preset in the monitoring hole; and $\varepsilon_{i}$ is the strain of the grating point and it can be calculated by $\varepsilon_{i}=\mu_{i} k$ while the wavelength can be determined by the ground fiber grating optic detector. 


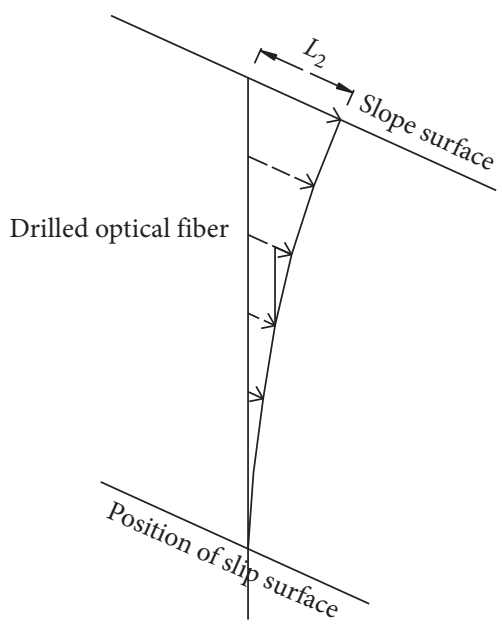

(a)

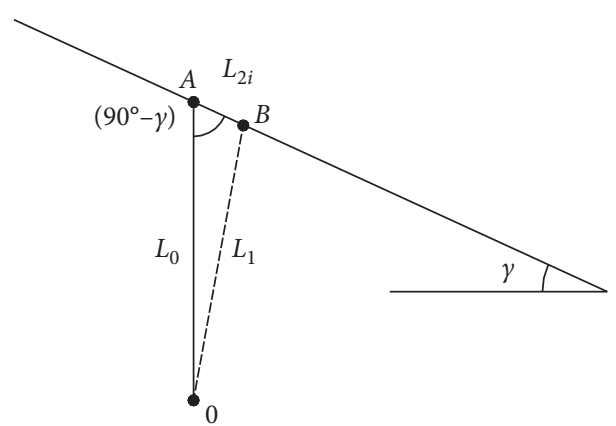

(b)

Figure 4: Schematic diagram of calculating the landslide internal deformation using fiber grating. (a) Calculation diagram of deflection curve. (b) Calculation diagram of deflection unit.

\section{Experiment}

3.1. Overview of the Toudu Landslide. The Toudu landslide is located in the south slope of Jinfo Mountain, Chongqing, China. The overall terrain is high in the north and low in the south. The rear edge of the landslide body is $1035 \mathrm{~m}$ above sea level, the elevation at the shearing exit is $900 \mathrm{~m}$, and the relative height difference of the landslide area is about $135 \mathrm{~m}$. The terrain slope is between $10^{\circ}$ and $25^{\circ}$, and the overall slope is $13^{\circ}$. The right side of the landslide is bounded by the natural gully, the left side is bounded by the ridge, the trailing edge is bounded by the steep junction, and the surrounding crack can be obviously observed. The surface of the Toudu landslide mainly includes terraced arable land, houses, and a small amount of forest land (as shown in Figure 5).

The landslide body is the pile-up body generated by the breaking down of the rock body in the upper part of the landslide and its accumulating in the middle and lower part of the slope and at the foot of the slope. In the early stage, the pileup body blocked the original river channel. Under the longterm impact and erosion of the river channel, the soil that blocked the river channel and broke down the pile-up body was washed out, and the soil and rock at the slope foot were hollowed out, forming large-pore rock stack layer. The landslide dam at the slope foot gradually collapsed under the gravity of the middle and upper part of the slope, which caused the whole slope to deform and landslide again. After experiencing the deformation process in multiple stages, the bedrock of the current stage was deeply buried, forming the slope composed of a silty soil layer and a thin layer of silty clay. Then, the Toudu landslide slid again after the rain, forming a higher water level.

Through geological exploration, it was found that the landslide had multiple layers suspected to be potential slip surfaces controlled by different rock-soil interfaces, as shown in Figure 6, which are labeled as slip surfaces $S 1, S 2$, $S 3, S 4$, and $S 5$, respectively. The potential slip surface $S 1$ is the interface between the surface silty clay and the lower rock layer; the potential slip surfaces $S 2$ and $S 4$ are the interface between the second silty clay layer and the lower rock layer; the potential slip surfaces $S 3$ and $S 5$ are the interface between the silty clay and the lower shale layer. As a consequence, geological workers cannot locate the main slip interfaces by geological surveys, drilling, or other means.

In this research, the FBG sensing technology was used to monitor the landslide internal deformation, thereby locating the position of the slip surface and exploring the mechanism of landslide internal deformation. Considering the deformation distribution characteristics of the entire landslide body, four monitoring holes were drilled in each greatly deformed position of the landslide, that is, middle-upper, middle, and lower-middle positions of the landslide, which are labeled as $D 1, D 2, D 3$, and $D 4$. The details are presented in Figure 6. D1, D3, and $D 4$ penetrated the potential slip surface of each layer and penetrated into the bedrock. Limited by the construction conditions, D2 only penetrated the potential slip surface $S 1$ and did not penetrate into the bedrock, as shown in Figure 6.

In order to verify the rationality of the proposed monitoring method and the formula with respect to landslide internal deformation, multiple monitoring points of surface displacement were set on the ground surface, and the surface monitoring points corresponding to $D 1, D 2, D 3$, and D4 were points $5,12,17$, and 18 .

3.2. Installation of Monitoring Equipment. In the experiment, the FGB monitoring equipment for landslide internal deformation mainly includes two parts: the data acquisition equipment and the FBG sensor. The data acquisition equipment is a convenient and dense quasidistributed optical fiber strain gauge. The FBG sensor adopts a conventional grating with point string, and the point spacing $L_{0}$ is $1.0 \mathrm{~m}$. The center characteristic wavelengths of the grating points are $1531 \mathrm{~nm}, 1543 \mathrm{~nm}$, and $1555 \mathrm{~nm}$, respectively, and the strain range is the wavelength difference $\Delta \lambda=8 \mathrm{~nm}$.

In the experiment, the fiber grating should be installed and constructed in a reasonable manner, which is very important for 


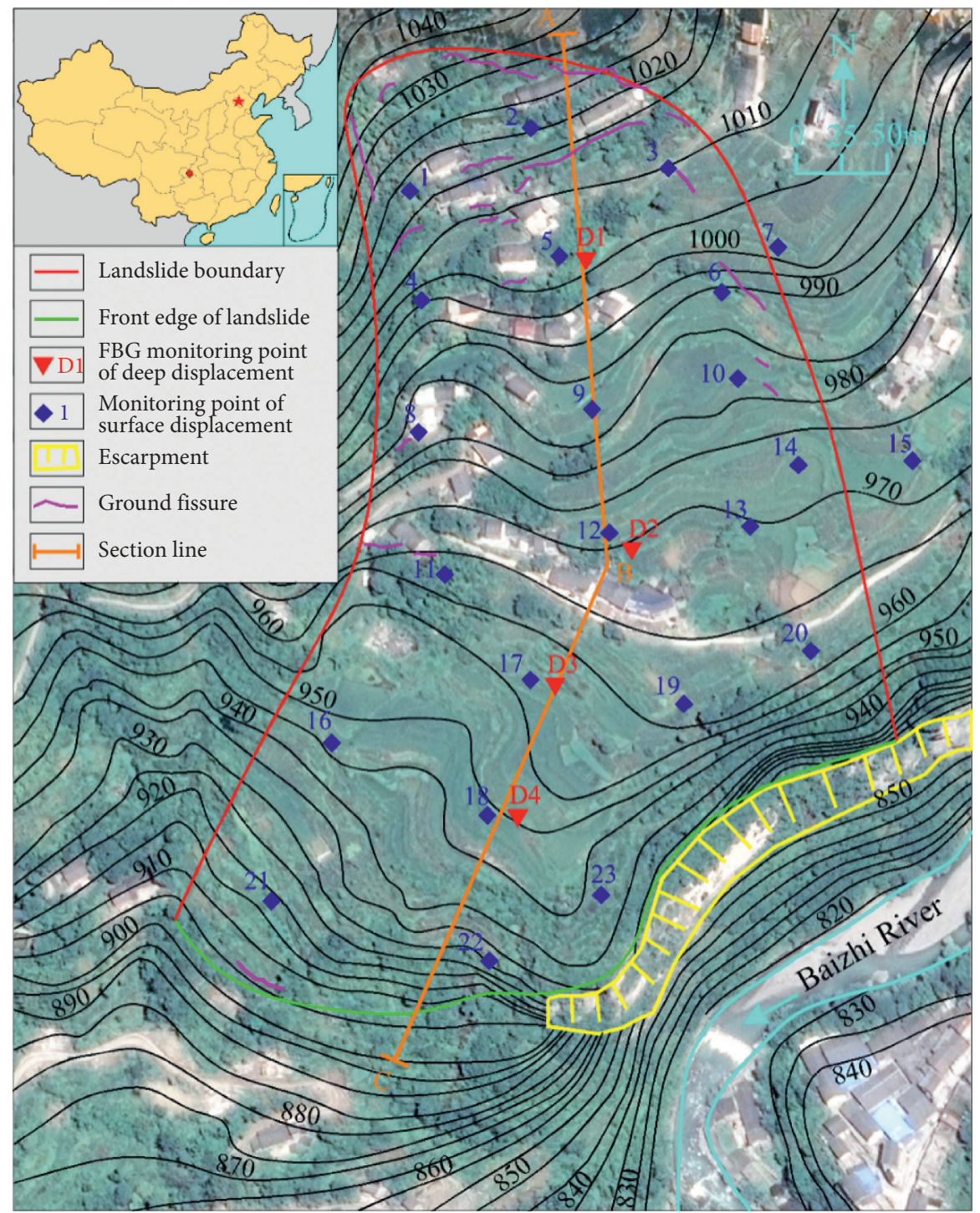

Figure 5: Layout of the Toudu landslide monitoring.

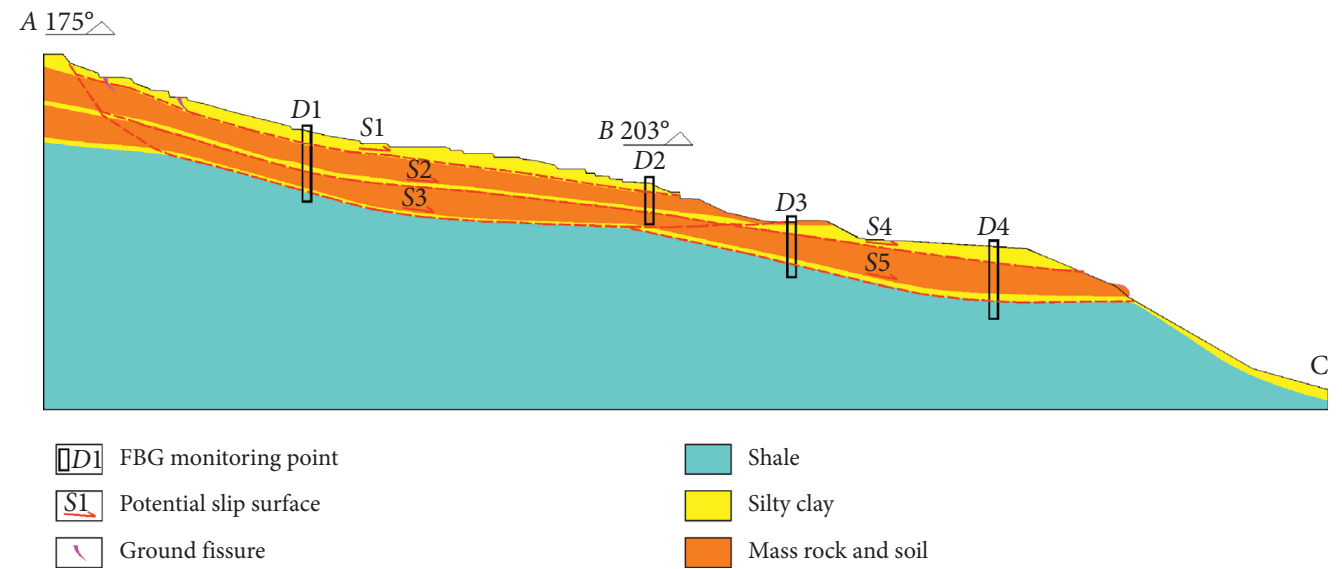

FIgURE 6: Cross-section of the monitoring of internal deformation for the Toudu landslide. 
the monitoring work. The installation process is shown in Figures 7 and 8. In order to coordinate the fiber sensing structure with the rock-soil body deformation, we used highstrength PVC tube as the conversion medium, fixed the fiber sensing structure to the high-strength PVC tube, and then embed the PVC tube in the drilled hole of landslide. The specific operation is as follows: (1) fiber grating sensor was pasted on the high-strength PVC tube with AB-type epoxy resin glue. After the pasting, the surface was sealed with cloth-based tape to isolate the water. (2) The monitoring holes were the holes that were just drilled for geological survey, with a diameter of $110 \mathrm{~mm}$ for each hole. The PVC tube with the fiber grating sensor was affixed vertically to the center of the hole, as shown in Figures 8 and 9. Then, the grouting filler was used to fill the hole. The clay and silty clay at site are hard to be compacted during backfilling process. For a better compaction and formation of dense structure, further, the excellent coupling effect between the equipment and the backfill materials, the dried and loose sand is adopted as the backfill materials. During sand backfilling, the sand is cautiously poured into the hole using a funnel with flow rate carefully controlling at $0.1 \mathrm{~L} / \mathrm{s}$ ). Then water is slowly injected into the sand filled hole, which will result in redistribution of the sand particles and effectively fill the gaps in the hole. Thereby ensuring that the sand could be deposited from bottom to top until it was filled. As a result, the fiber grating tube was in good contact with the landslide body, playing the role of deformation coordination.

In order to eliminate the influence of the temperature on the FBG sensor, a temperature-compensated FBG sensor was installed along the inside of the PVC tube, and it could freely retract from the tube (as shown in Figures 7 and 8). Therefore, during the landslide deformation process, the strain of the temperature-compensated fiber grating would not be caused by the deformation of the tube. The data in this paper has already been corrected with consideration of the temperature compensation. The FBG sensor was firmly pasted to high-strength PVC tube, and the flow of water had little effect on the deformation of the PVC tube. Therefore, the flow of water had little effect on the strain of the FBG sensor, which could be ignored.

In order to test the deformation performance of the FBG sensor and the PVC tube and to calibrate the value of the constant $k, \mathrm{AB}$ glue was used to attach the FBG sensor to the PVC tube, and then a tensile test is performed on the PVC tube under laboratory conditions. The deformation of the FBG sensor and the PVC tube is monitored simultaneously, and the constant $k$ is calibrated to be $845\left(\mathrm{~mm}^{-1}\right)$.

The installation parameters of the equipment at each monitoring hole are shown in Table 1 . The number of grating points in $D 1, D 2, D 3$, and D4 are $34,22,30$, and 34 , respectively.

\section{Results Analysis}

The data of landslide internal deformation at the four monitoring points were collected in an irregular manner from December 3, 2018, to February 19, 2019. The collected data were used for analyzing the position of slip surface and the mechanism of landslide internal deformation.

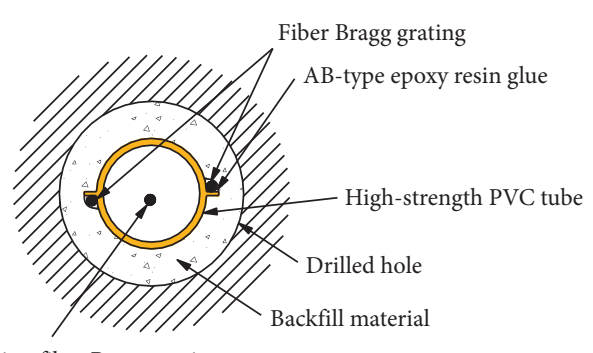

Temperature compensation fiber Bragg grating

FIgURE 7: Schematic diagram of the cross-section of the monitoring hole installed with fiber grating.

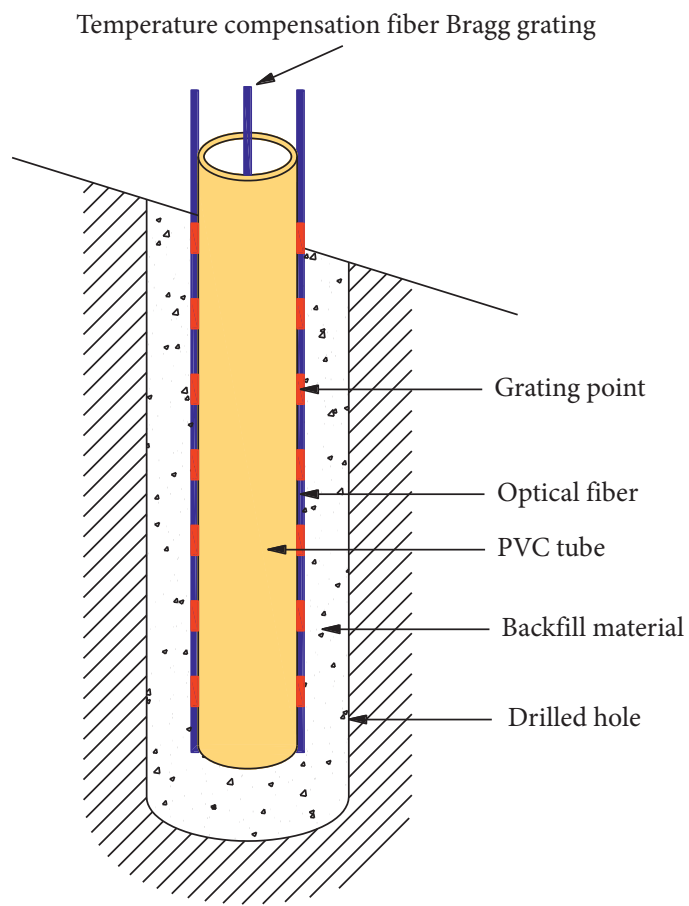

FIGURE 8: Schematic diagram of the equipment installation for the monitoring of landslide internal deformation using fiber grating.

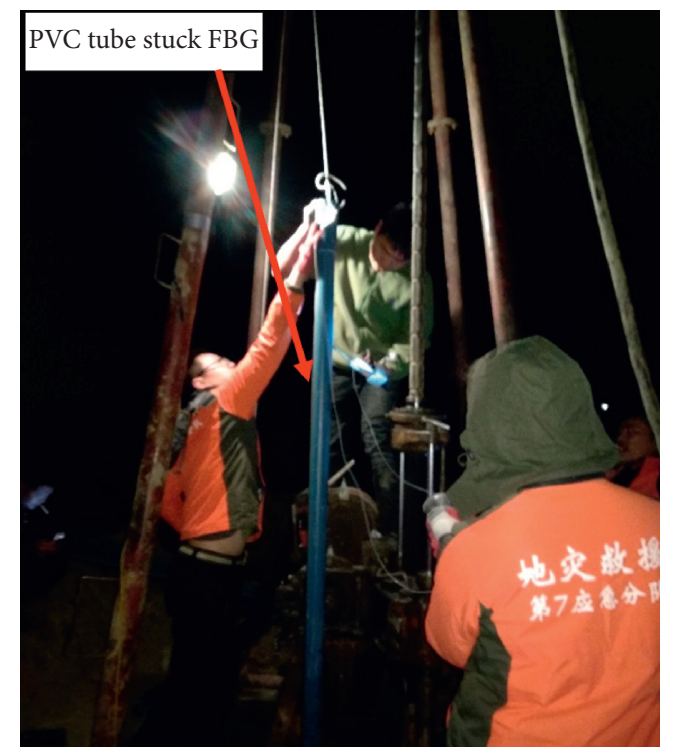

FIgURE 9: Field installation of fiber grating monitoring equipment. 
TABLE 1: Monitoring hole information of fiber grating monitoring.

\begin{tabular}{lcc}
\hline Monitoring points & Thickness of drilled soil layer $(\mathrm{m})$ & Number of grating points \\
\hline D1 & 29.6 & 34 \\
$D 2$ & 28.2 & 22 \\
$D 3$ & 28.5 & 30 \\
$D 4$ & 30.7 & 34 \\
\hline
\end{tabular}

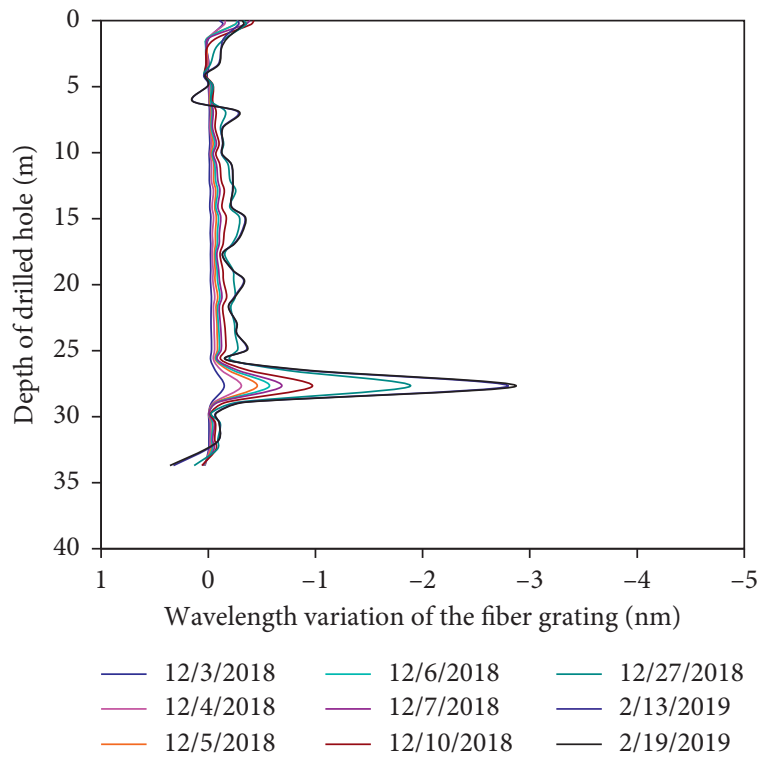

Figure 10: Wavelength of fiber grating at $D 1$.

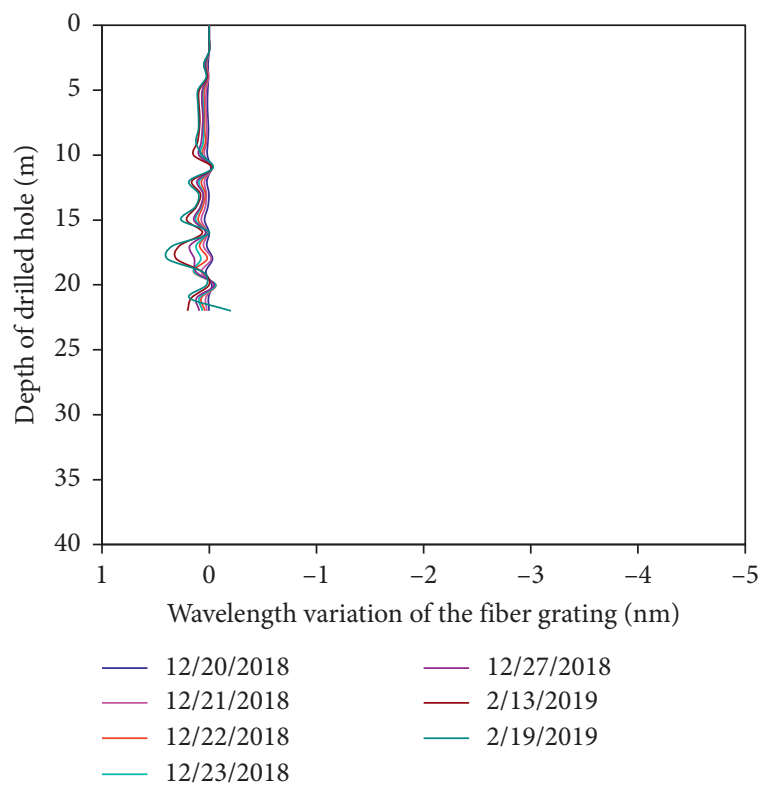

Figure 11: Wavelength of fiber grating at D2.

4.1. Recognition of Landslide Slip Surface. In the experiment, we collected the center wavelength of the reflected wave along the drilling depth at four monitoring holes $D 1, D 2, D 3$, and $D 4$. The results are shown in Figures 10-13. It can be seen from the figures that, the central wavelength at $D 1, D 2$, $D 3$, and D4 gradually increases with depth increasing, and

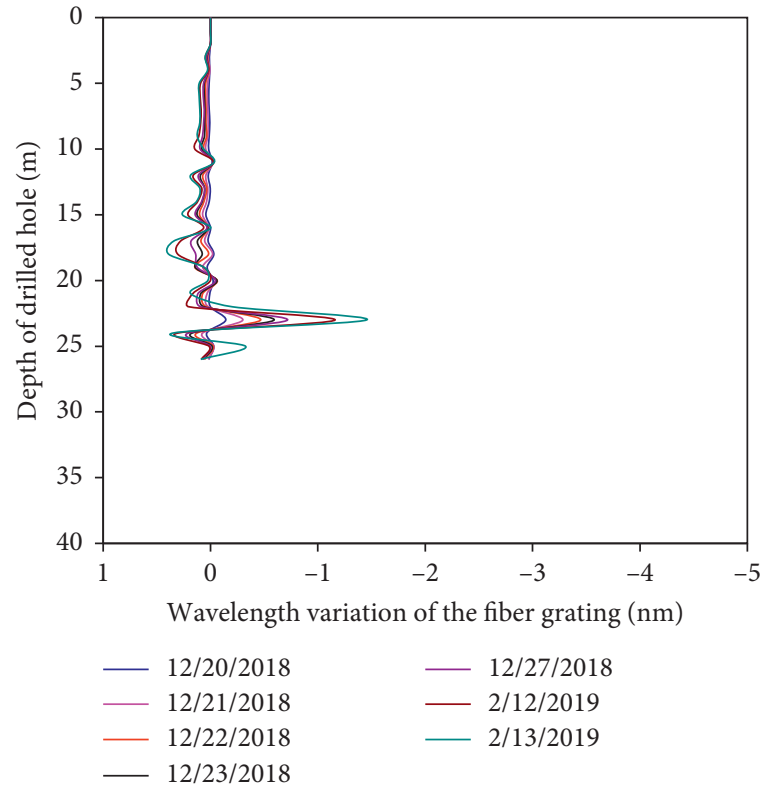

Figure 12: Wavelength of fiber grating at D3.

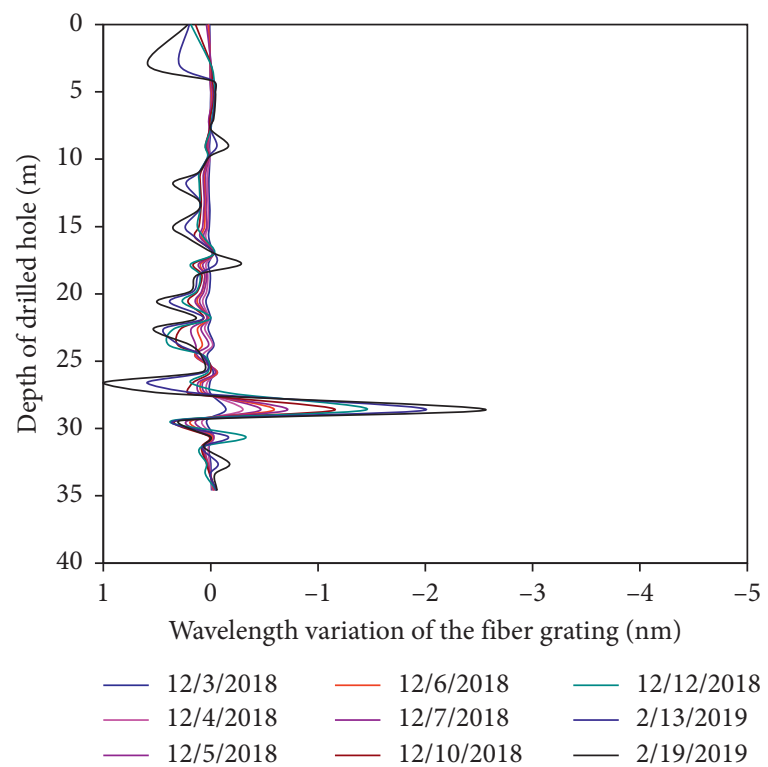

Figure 13: Wavelength of fiber grating at $D 4$.

after reaching a certain depth, the wavelengths of $D 1, D 3$, and $D 4$ have a sudden change at the positions of $27.7 \mathrm{~m}, 24.0 \mathrm{~m}$, and $28.6 \mathrm{~m}$, respectively. This indicates that at these positions, the fiber grating had large strain and the rock-soil body was also deformed greatly. So, it could be determined that the positions 


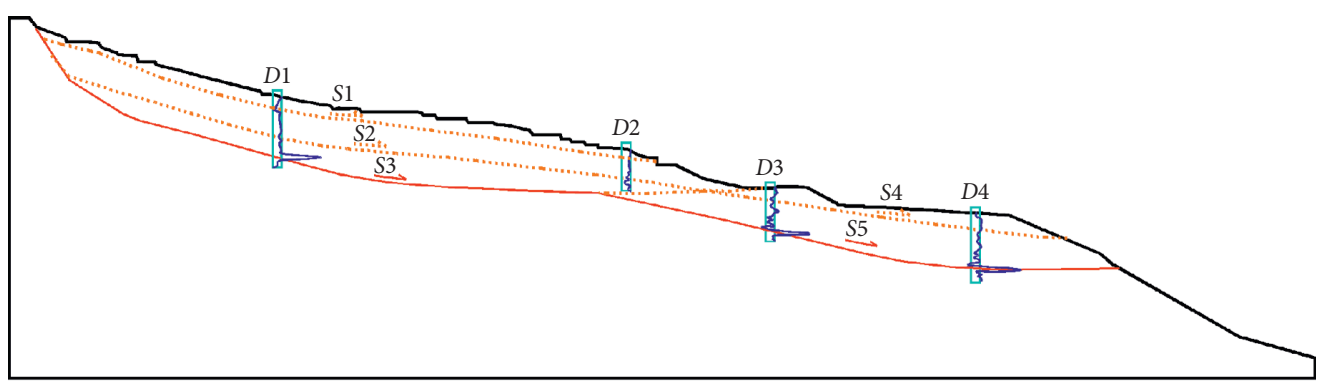

$\square D 1$ FBG monitoring point

S1.... Excluded potential slip surface

Determined slip surface

Figure 14: Cross-section of the monitoring of internal deformation for the Toudu landslide.

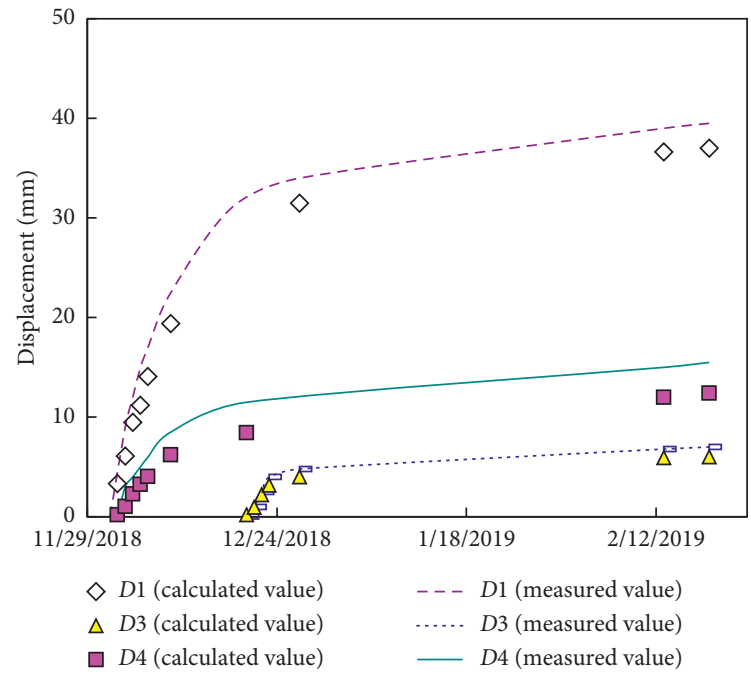

FIGURE 15: Comparison of the surface displacement calculated by the proposed formula and the measured values.

were exactly the positions of the main slip surfaces of the landslide. No abrupt change in wavelength was observed at D2, indicating that D2 did not penetrate the main slip surface of the landslide. At the same time, from the comparison and analysis of the monitoring data collected in multiperiods, the central characteristic wavelengths of the grating points at $D 1, D 2, D 3$, and $D 4$ increased with time increasing, indicating that the landslide continuously produced slow sliding deformation from the day of starting monitoring.

By integrating the data at the four holes, the main slip surfaces of the landslide could be obtained, as shown in Figure 14. It could be seen from the figure that the landslide mainly slid deep along the interface between the shale and the silty clay. With the potential slip surfaces $S 1, S 2$, and $S 4$ excluded, it was determined that the main slip surfaces were $S 3$ and S5.

4.2. Landslide Internal Deformation. According to the formula for calculating the displacement of any mass point in the landslide with respect to the strain of the fiber grating, the surface displacements of $D 1, D 3$, and $D 4$ could be calculated and were then compared with the surface displacement of monitoring points 5,17 , and 18 adjacent to the

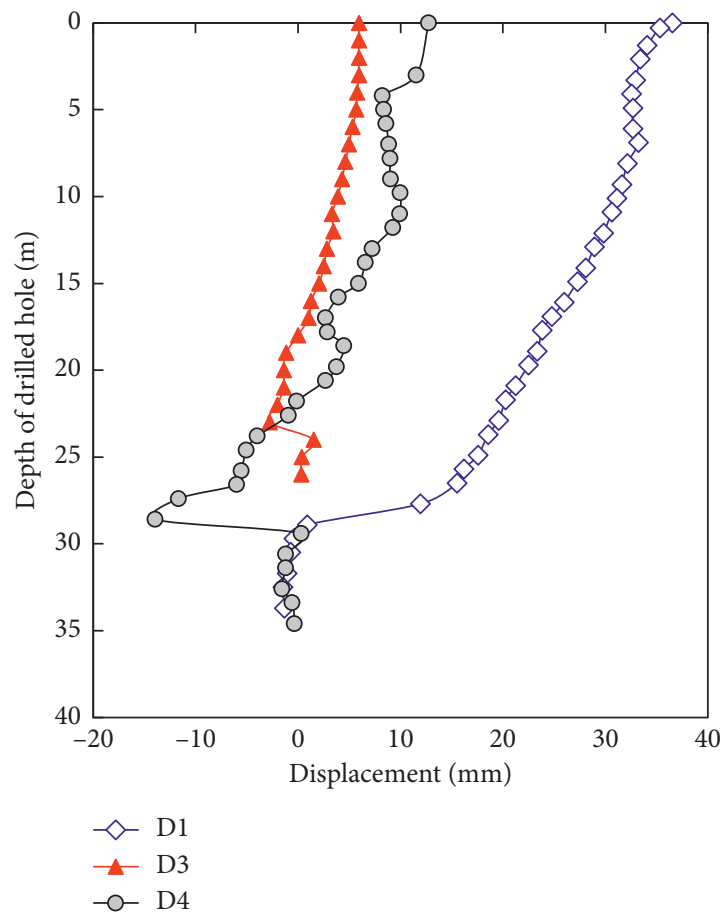

FIGURE 16: Internal deformation of landslide.

three points (as shown in Figure 15). It could be seen from Figure 15 that the surface displacements obtained by the formula were basically consistent with the measured ones, and the measured values were slightly larger than the calculated values, indicating the rationality of the formula.

According to the formula for calculating the displacement of any mass point in the landslide with respect to the strain of the fiber grating, the displacements of internal mass points in the landslide at $D 1, D 3$, and $D 4$ could be calculated (the data were collected on February 19, 2019), as shown in Figure 16. The monitoring point $D 1$ was located at the trailing edge of the landslide, where the rock-soil mass point above the slip surface moved down along the slope as a whole, and the displacement value gradually increased along the sliding surface. The monitoring point $D 4$ was located at the front edge of the landslide, where the rock-soil mass point above the slip surface moved down along the slope as a 
whole, but a disturbance displacement occurred along the slope on the slip surface. The monitoring point D3 was located in the lower-middle part of the landslide, where the rock-soil mass point above the slip surface moved down along the slope as a whole, but a disturbance displacement occurred along the slope on the slip surface, and its value was smaller than that at $D 4$.

According to the internal deformation mechanism of $D 1$, $D 3$, and $D 4$, it could be preliminarily determined that the landslide was a thrust load-caused landslide caused by rainfall-induced instability of the trailing edge of the landslide.

\section{Conclusions}

Taking the Toudu landslide as an example, this paper presents the application of FBG to the in situ monitoring of landslide internal deformation. The conclusions are summarized as follows:

(1) Based on the FBG sensing technology, this research locates the position of the main slip surface of the landslide and proposes the slip surface recognition method.

(2) Based on the deflection curve of landslide internal deformation, the formula for calculating the landslide internal deformation with respect to fiber grating strain is proposed. The rationality of the formula is verified by the comparison of calculated displacement values and measured ones. The internal deformation of the Toudu landslide is analyzed to clarify the formation mechanism of the Toudu landslide.

\section{Data Availability}

The data used to support the findings of this study are available from the corresponding author upon request.

\section{Conflicts of Interest}

The authors declare that they have no conflicts of interest.

\section{Acknowledgments}

The authors are grateful to the Performance, Motivation and Guidance Special Project of Chongqing Scientific Research Institutions (Project no. cstc2019jxj100006) and the Science and Technology Planning Project of Chongqing Land Resources and Housing Management (Project no. KJ-2019002).

\section{References}

[1] K. O. Hill, Y. Fujii, D. C. Johnson, and B. S. Kawasaki, "Photosensitivity in optical fiber waveguides: application to reflection filter fabrication," Applied Physics Letters, vol. 32, no. 10, pp. 647-649, 1978.

[2] A. Kawasaki, T. F. Morse, and F. Mendez, "Applications of embedded optical fiber sensors in reinforced concrete buildings and structures," in Proceedings of the SPIE Fiber Grating Smart Structures and Skins, pp. 60-69, Sydney, Australia, 1990.
[3] G. Meltz, W. W. Morey, and W. H. Glenn, "Formation of Bragg gratings in optical fibers by a transverse holographic method," Optics Letters, vol. 14, no. 15, pp. 823-825, 1989.

[4] R. L. Idriss, "Monitoring of a smart bridge with embedded sensors during manufacturing, construction and service," in Proceedings of the 3rd International Workshop on Structural Health Monitoring, pp. 604-613, Stanford, CA, USA, September 2001.

[5] J. Ou and W. U. Z. Zhouzhi, "Intelligent monitoring of Heilongiiang Hulanriver bridge based on FBGS," China Civil Engineering Journal, vol. 37, no. 1, pp. 45-50, 2004.

[6] P. Kronenberg, N. Casanova, D. Inaudi et al., "Dam monitoring with fiber gratings deformation sensors," in Proceedings of the SPIE, Smart Structures and Materials, pp. 2-11, Kissimmee, FL, USA, June 1997.

[7] E. Udd, M. Kunzler, M. H. Layor et al., "Fiber grating systems for traffic monitoring," in Proceedings of the SPIE, Health Monitoring and Management of Civil Infrastructure Systems, pp. 510-514, Newport Beach, CA, USA, March 2001.

[8] T. H. T. Chan, L. Yu, H. Y. Tam et al., "Fiber Bragg grating sensors for structural health monitoring of Tsing Ma bridge: background and experimental observation," Engineering Structures, vol. 28, no. 5, pp. 648-659, 2006.

[9] B. Ni, X. Xu, D. I. Wang et al., "Study on BOTDR based distributed optical fiber strain measurement for tunnel health diagnosis," Chinese Journal of Rock Mechanics and Engineering, vol. 24, no. 15, pp. 26-28, 2005.

[10] D. Inaudi, "Application of optical fiber sensor in civil structural monitoring," in Proceedings of SPIE, Fiber Grating Sensors and Their Application, pp. 1-10, Calcutta, India, 2001.

[11] F. Tavenas, C. Mieussens, and F. Bourges, "Lateral displacements in clay foundations under embankments," Canadian Geotechnical Journal, vol. 16, no. 3, pp. 532-550, 1979.

[12] M. Hisham, J. Peter, S. Kenichi et al., "Distributed optical fiber strain sensing in a secant piled wall," in Proceedings of the Seventh International Symposium on Filed Measurements in Geomechanics, pp. 22-31, Boston, MA, USA, September 2007.

[13] B. Shi, H. Xu, D. Zhang et al., "Feasibility study on application of BOTDR to health monitoring for large infrastructure engineering," Chinese Journal of Rock Mechanics and Engineering, vol. 23, no. 1, pp. 493-499, 2004.

[14] Y. Shi, Q. Zhang, and X. Meng, "The application of distributed optical fiber sensing technology in landslide monitoring," Journal of Jilin University: Earth Science, vol. 38, no. 5, pp. 820-824, 2008.

[15] Y. Liu, H. Sun, Y. Shang et al., "Study on the fixation technique of optical fiber base on BOTDR," Chinese Journal of Sensors and Actuators, vol. 23, no. 9, pp. 1353-1358, 2010.

[16] J. Liu, B. Shi, D. Zhang et al., "Experimental study of foundation pit monitoring using BOTDR-based on distributed optical fiber sensor," Chinese Journal of Rock Mechanics and Engineering, vol. 27, no. 7, pp. 1224-1228, 2006.

[17] B. Wang and B. Shi, "Distributed fiber monitoring test and its application to slope deformation," Journal of Disaster Prevention and Mitigation Engineering, vol. 30, no. 1, pp. 28-34, 2010.

[18] Y. Liu, Y. Shang, and Y. U. Yang, "Application of surface deformation monitoring of slope using BOTDR technology," Journal of Jilin University: Earth Science, vol. 41, no. 3, pp. 777-783, 2011.

[19] H. Pei, P. Cui, J. Yin et al., "Monitoring and warning of landslides and debris flows using an optical fiber sensor technology," Journal of Mountain Science, vol. 8, no. 5, pp. 728-738, 2011. 
[20] H.-Hu Zhu, B. Shi, J.-F. Yan, J. Zhang, C.-C. Zhang, and B.-J. Wang, "Fiber Bragg grating-based performance monitoring of a slope model subjected to seepage," Smart Materials and Structures, vol. 23, no. 9, Article ID 095027, 2014.

[21] D. Zhang, Q. Xu, A. Bezuijen, G. Zheng, and H. Wang, "Internal deformation monitoring for centrifuge slope model with embedded FBG arrays," Landslides, vol. 14, no. 1, pp. 407-417, 2016.

[22] H. Zheng, D. Zhang, Y. Gao et al., "Strain distribution based geometric models for characterizing the deformation of a sliding zone," Engineering Geology, vol. 263, Article ID 105300, 2019.

[23] H. Fang, D. Zhang, Z. Song, and B. Shi, "BOTDA based investigation on the effects of closure strips in bottom plate during the construction of navigation lock," Measurement, vol. 117, pp. 67-72, 2018.

[24] Y. Shi, B. Shi, D. Zhang, H. Tong, G. Wei, and H. Xu, "Internal deformation monitoring of slope based on BOTDR," Journal of Sensors, vol. 2016, pp. 1-8, 2016.

[25] H.-H. Wei, Z.-Y. Wang, B. Shi, and J. Kam-Wing Wong, "Feasibility study of strain based stability evaluation of locally loaded slopes. insights form physical and numerical modeling," Engineering Geology, vol. 208, pp. 39-50, 2016.

[26] H. Pei, J. Yin, and H. Zhu, "In-situ monitoring of didplacements and stability evaluation of slope based on fiber Bragg grating sensing technology," Chinese Journal of Rock Mechanics and Engineering, vol. 29, no. 8, pp. 1570-1576, 2010.

[27] Y. Zheng, Z.-W. Zhu, Q.-X. Deng, and F. Xiao, “Theoretical and experimental study on the fiber Bragg grating-based inclinometer for slope displacement monitoring," Optical Fiber Technology, vol. 49, pp. 28-36, 2019. 